\title{
Naive Planes as Discrete Combinatorial Surfaces
}

\author{
Yukiko Kenmochi ${ }^{1}$ and Atsushi Imiya ${ }^{2}$ \\ 1 School of Information Science, Japan Advanced Institute of Science and Technology \\ kenmochi@jaist.ac.jp \\ 2 Department of Information and Image Sciences, Chiba University \\ imiya@ics.tj.chiba-u.ac.jp
}

\begin{abstract}
An object of interest is digitized if we acquire its 3-dimensional digital images by using techniques such as computerized tomographic imaging. For recognition or shape analysis of such digitized objects, we need the study of 3-dimensional digital geometry and topology. In this paper, we focus on one of the simplest geometric objects such as planes and study their geometric and topological properties which are expressed by using an algebraic method.
\end{abstract}

\section{Introduction}

In this paper, we deal with the geometric and topological properties of digitized objects which are expressed by using an algebraic method. An object of interest is digitized if we acquire its 3-dimensional digital images by using techniques such as computerized tomographic imaging. For recognition and shape analysis of such digitized objects, topological constraints such as topological equivalence in use of Euler characteristics [1], skeletons [2], combinatorial manifolds [4], etc. are used as well as geometric constraints which are given by algebraic equations/inequations. In this paper, we focus on one of the simplest geometric objects such as planes and study not only their digital geometry but their digital topology for 3-dimensional computer imagery.

In the context of digital geometry, an algebraic approach for the study of geometric properties of planes in an integer lattice space has been proposed in $[3,4,5,6,7]$. They have defined a naive/standard plane which is a set of integer lattice points and proposed the theory using algebraic properties of a lattice space. The algebraic properties such as local configurations of points in naive/standard planes have been derived by their algebraic approach.

On the other hand, we have proposed an approach based on combinatorial topology [8,9] for the definition and construction of topological planes in an integer lattice space; they are called discrete planar surfaces and constructed by applying our boundary extraction algorithm [8]. In this paper, we clarify the relations between our discrete planar surfaces and naive planes such that our discrete planar surface for 18- or 26-neighborhood system is a triangulation of a naive plane in the aspect of combinatorial topology [10]; in [4], a triangulation has been introduced in a similar approach for a standard plane, but not for a naive plane. By seeing the topological structures of naive planes as discrete 
planar surfaces, we show their geometric and topological properties in an integer lattice space, which can be derived from the algebraic properties of naive planes.

\section{Definition of Naive Planes}

Let $\mathbf{R}$ be the set of real numbers; $\mathbf{R}^{3}$ denotes the 3 -dimensional Euclidean space. A plane $\mathbf{P}$ in $\mathbf{R}^{3}$ is defined by

$$
\mathbf{P}=\left\{(x, y, z) \in \mathbf{R}^{3}: a x+b y+c z+d=0\right\}
$$

where $a, b, c, d$ are real numbers. Let $\mathbf{Z}$ be the set of real numbers; $\mathbf{Z}^{3}$ denotes the set of lattice points whose coordinates are all integers. We introduce the definition of planes in $\mathbf{Z}^{3}$ based on algebraic approach [5]. The naive plane is defined with respect to $\mathbf{P}$ by

$$
\mathbf{N P}=\left\{(x, y, z) \in \mathbf{Z}^{3}: 0 \leq a x+b y+c z+d<w\right\}
$$

where $w=\max \{|a|,|b|,|c|\}$. The parameter $w$ is called the width of NP.

\section{Definition of Discrete Planar Surfaces}

\subsection{Definition of Discrete Combinatorial Surfaces}

In this subsection, we introduce the definition of surfaces in $\mathbf{Z}^{3}$ based on the approach of combinatorial topology [10]. In $\mathbf{Z}^{3}$ we define three different neighborhoods of a lattice point $\boldsymbol{x}=(i, j, k)$ as

$$
\mathbf{N}_{m}(\boldsymbol{x})=\left\{(p, q, r) \in \mathbf{Z}^{3}:(i-p)^{2}+(j-q)^{2}+(k-r)^{2} \leq t\right\}
$$

for $m=6,18,26$ corresponding to $t=1,2,3$. They are called 6 -, 18 - and 26-neighborhoods, respectively. Depending on each neighborhood, we define elements of 1-dimensional curves and 2-dimensional surfaces in $\mathbf{Z}^{3}$. These elements are called 1- and 2-dimensional discrete simplexes and abbreviated as 1and 2-simplexes, respectively. Suppose we define 0-dimensional discrete simplexes, which are called 0-simplexes, as isolated points in $\mathbf{Z}^{3}$. Then 1 - and 2simplexes are defined recursively as follows.

Definition 1. An n-simplex for $n=1,2$ is defined as a set of $k$ points in $\mathbf{Z}^{3}$, $\left[\boldsymbol{x}_{1}, \boldsymbol{x}_{2}, \ldots, \boldsymbol{x}_{k}\right]=\left\{\boldsymbol{x}_{1}, \boldsymbol{x}_{2}, \ldots, \boldsymbol{x}_{k}\right\}$ so that the closed convex hull of $\boldsymbol{x}_{1}, \boldsymbol{x}_{2}, \ldots, \boldsymbol{x}_{k}$ is one of $n$-dimensional minimum nonzero regions in $\mathbf{R}^{3}$ which are bounded by the closed convex hulls of $(n-1)$-simplexes.

According to Definition 1, a 1-simplex consists of two neighboring points in $\mathbf{Z}^{3}$. The configurations of those two neighboring points depend on the neighborhood systems as shown in the first line of Table 1. A 2-simplex is defined as a set of points whose closed convex hull is bound by a set of the closed convex 
Table 1. Each $n$-simplexes for $n=1,2$ is defined as a set of points in $\mathbf{Z}^{3}$ for the 6-, 18- and 26-neighborhood systems. All $n$-simplexes are obtained by rotation and translation of those in the table

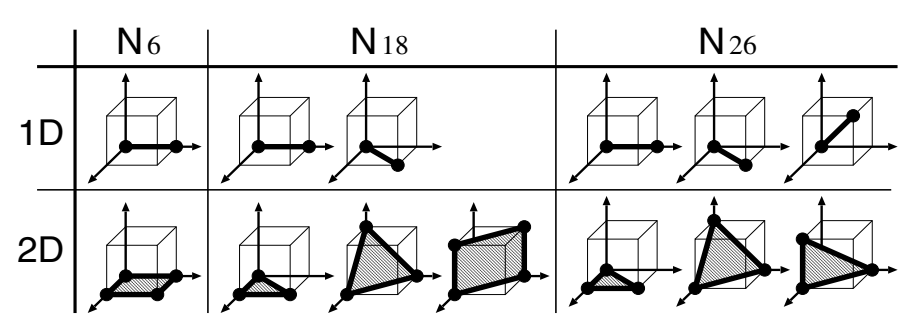

hulls of 1-simplexes and holds a 2-dimensional minimum nonzero area. All 2simplexes for each neighborhood system are shown in the second line of Table 1. The constructive definitions of 1- and 2-simplexes are presented in [8].

If an $n_{1}$-simplex is a subset of an $n_{2}$-simplex where $n_{1}<n_{2}$, the $n_{1}$-simplex is called a face of the $n_{2}$-simplex; it is also called an $n_{1}$-face. For instance, a 2 simplex for the 26-neighborhood system has three 0 -faces and three 1-faces. A set of all faces included in a discrete simplex $[a]=\left[\boldsymbol{x}_{1}, \boldsymbol{x}_{2}, \ldots, \boldsymbol{x}_{k}\right]$ is denoted by face $([a])$. Let the closed convex hull of $k$ points, $\boldsymbol{x}_{1}, \boldsymbol{x}_{2}, \ldots, \boldsymbol{x}_{k}$, be denoted by $\mathbf{C H}\left(\left\{\boldsymbol{x}_{1}, \boldsymbol{x}_{2}, \ldots, \boldsymbol{x}_{k}\right\}\right)$. The embedded discrete simplex is defined as

$$
\|a\|=\mathbf{C H}([a]) \backslash(\underset{[b] \in f a c e([a])}{\cup} \mathbf{C H}([b]))
$$

for any $n$-simplex $[a]$, and $\|a\|$ is called the embedded $n$-simplex of $[a]$. An $n$ simplex and the embedded $n$-simplex are clearly different since $[a]$ and $\|a\|$ are defined as sets of points in $\mathbf{Z}^{3}$ and $\mathbf{R}^{3}$, respectively.

Definition 2. A finite set $\mathbf{K}$ of discrete simplexes is called a discrete complex if it satisfies the following conditions: if $[a] \in \mathbf{K}$, face $([a]) \subseteq \mathbf{K}$; if $[a],[b] \in \mathbf{K}$ and $\|a\| \cap\|b\| \neq \emptyset$, then $[a]=[b]$.

The dimension of $\mathbf{K}$ is equal to the maximum dimension of discrete simplexes which belong to $\mathbf{K}$. Hereafter, we abbreviate $n$-dimensional discrete complexes to $n$-complexes as well as $n$-simplexes. Suppose that $\mathbf{K}$ is an $n$-complex. If there exist at least one $n$-simplex $[a] \in \mathbf{K}$ for every $s$-simplex $[b] \in \mathbf{K}$ such that $[b] \in$ face $([a])$ and $s<n, \mathbf{K}$ is called pure. If we can find a chain of discrete simplexes between two arbitrary elements $[c],[d] \in \mathbf{K},\left[c_{1}\right]=[c],\left[c_{2}\right], \ldots,\left[c_{k}\right]=[d]$, such that $\left[c_{i}\right]$ and $\left[c_{i+1}\right], i=1,2, \ldots, k-1$, has a common face in $\mathbf{K}, \mathbf{K}$ is called connected.

Definition 3. If a 2-complex $\mathbf{K}$ is pure and connected, $\mathbf{K}$ is a discrete combinatorial surface.

More discussion on discrete combinatorial surfaces in the sense of combinatorial topology is given in [8]. Note that discrete complexes and discrete combinatorial surfaces are constructed with respect to each neighborhood system. 
Table 2. A set $\partial \mathbf{I}_{m}^{+}(i, j, k)$ for each of eight possible configurations of black and white points in $\mathbf{C}(i, j, k), m=6,18,26$. In the table, we consider $\mathbf{P}$ such that $0 \leq a \leq b \leq c, c>0$. The configurations within parentheses are ignored for the construction of $\partial \mathbf{I}_{m}^{+}$because black points in such $\mathbf{C}(i, j, k)$ are regarded as 0 - or 1 -faces of 2-simplexes in the adjacent cubes of $\mathbf{C}(i, j, k)$

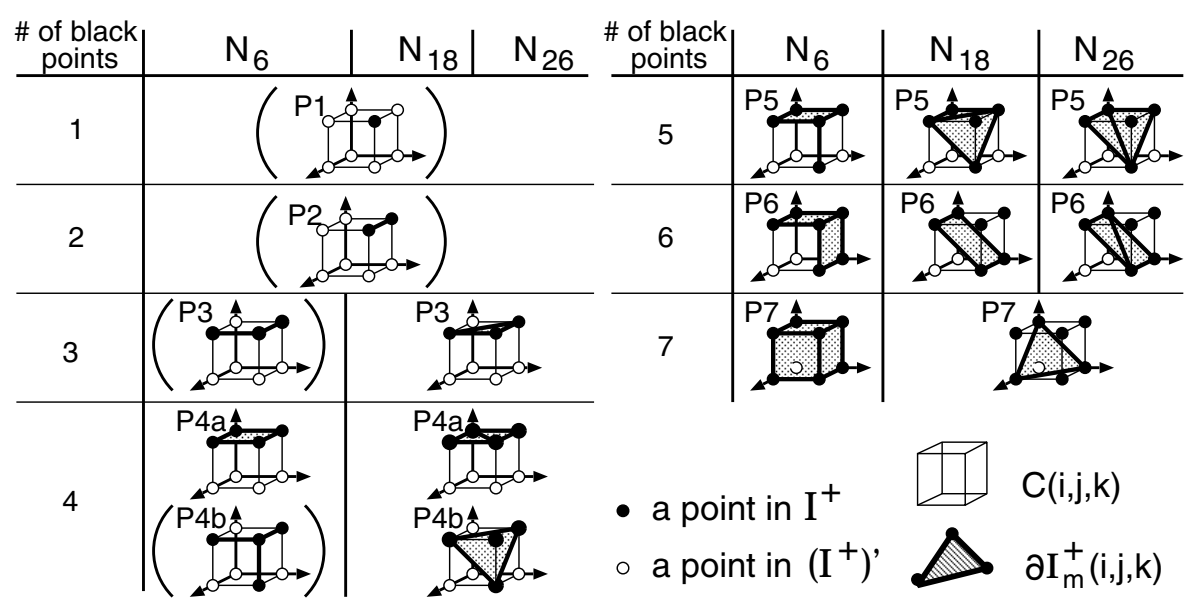

\subsection{Construction of Discrete Planar Surfaces}

The following two regions in $\mathbf{R}^{3}$ are separated by $\mathbf{P}$ :

$$
\begin{aligned}
& \mathbf{H}^{-}=\left\{(x, y, z) \in \mathbf{R}^{3}: a x+b y+c z+d \leq 0\right\}, \\
& \mathbf{H}^{+}=\left\{(x, y, z) \in \mathbf{R}^{3}: a x+b y+c z+d \geq 0\right\} .
\end{aligned}
$$

Obviously, we have

$$
\mathbf{H}^{-} \cap \mathbf{H}^{+}=\mathbf{P} .
$$

Just as $\mathbf{H}^{-}$and $\mathbf{H}^{+}$in $\mathbf{R}^{3}$, there are two regions in $\mathbf{Z}^{3}$, which are separated by $\mathbf{P}$ as follows:

$$
\begin{aligned}
& \mathbf{I}^{-}=\left\{(x, y, z) \in \mathbf{Z}^{3}: a x+b y+c z+d \leq 0\right\} \\
& \mathbf{I}^{+}=\left\{(x, y, z) \in \mathbf{Z}^{3}: a x+b y+c z+d \geq 0\right\}
\end{aligned}
$$

We say that $\mathbf{I}^{-}$and $\mathbf{I}^{+}$are the digitization of $\mathbf{H}^{-}$and $\mathbf{H}^{+}$, respectively. For both $\mathbf{I}^{-}$and $\mathbf{I}^{+}$, we can construct the boundaries which are discrete combinatorial surfaces with the $m$-neighborhood system for $m=6,18,26$, denoted by $\partial \mathbf{I}_{m}^{-}$and $\partial \mathbf{I}_{m}^{+}$, using the similar algorithm for boundary extraction [8]. Both $\partial \mathbf{I}_{m}^{-}$and $\partial \mathbf{I}_{m}^{+}$ are considered to be the digitization of $\mathbf{P}$ and called discrete planar surfaces with respect to $\mathbf{P}$. In this subsection, we present how to generate $\partial \mathbf{I}_{m}^{+}$from $\mathbf{I}^{+}$. The same procedure can be applied to generate $\partial \mathbf{I}_{m}^{-}$if $\mathbf{I}^{+}$and $\partial \mathbf{I}_{m}^{+}$are replaced by $\mathbf{I}^{-}$and $\partial \mathbf{I}_{m}^{-}$, respectively. 


\section{Algorithm 1.}

input: $\mathbf{I}^{+}$.

output: $\partial \mathbf{I}_{m}^{+}$.

begin

1. Points in $\mathbf{I}^{+}$and the complement $\left(\mathbf{I}^{+}\right)^{\prime}=\mathbf{Z}^{3} \backslash \mathbf{I}^{+}$are assigned black and white points, respectively;

2. in any unit cubic region

$$
\mathbf{C}(i, j, k)=\left\{(x, y, z) \in \mathbf{Z}^{3}: i \leq x \leq i+1, j \leq y \leq j+1, k \leq z \leq k+1\right\}
$$

such that $\mathbf{C}(i, j, k) \cap \mathbf{I}^{+} \neq \emptyset$ and $\mathbf{C}(i, j, k) \cap\left(\mathbf{I}^{+}\right)^{\prime} \neq \emptyset$, the black and white points have either of eight different configurations as shown in Table 2;

3. for each $\mathbf{C}(i, j, k), \partial \mathbf{I}_{m}^{+}(i, j, k)$ is obtained as a set of 2-simplexes and their faces by referring to Table 2;

4. obtain

end

$$
\partial \mathbf{I}_{m}^{+}=\underset{(i, j, k) \in \mathbf{Z}^{3}}{\cup} \partial \mathbf{I}_{m}^{+}(i, j, k) .
$$

The next theorem is derived; the proof is given in [9].

Theorem 1. Each $\partial \mathbf{I}_{m}^{+}$(resp. $\partial \mathbf{I}_{m}^{-}$) for $m=6,18,26$ obtained from $\mathbf{I}^{+}$(resp. $\mathbf{I}^{-}$) by Algorithm 1 is a discrete combinatorial surface and called a discrete planar surface.

\section{Relations between Naive Planes and Discrete Planar Surfaces}

Let $\mathbf{B}_{m}^{+}$be the set of all lattice points in $\partial \mathbf{I}_{m}^{+}$for $m=6,18,26$, such that

$$
\mathbf{B}_{m}^{+}=\underset{[a] \in \partial \mathbf{I}_{m}^{+}}{\cup}[a] .
$$

Then, the following lemma is derived.

Lemma 1. For any plane $\mathbf{P}$, the inclusion and equality relations

$$
\mathbf{B}_{6}^{+} \supseteq \mathbf{B}_{18}^{+}=\mathbf{B}_{26}^{+}
$$

hold.

Proof. Using $\mathbf{C}(i, j, k)$ of (10), for each $m$, we define

$$
\mathbf{B}_{m}^{+}(i, j, k)=\mathbf{B}_{m}^{+} \cap \mathbf{C}(i, j, k)
$$

which is a subset of $\mathbf{B}_{m}^{+}$. Let us compare a triplet of $\mathbf{B}_{m}^{+}(i, j, k), m=6,18,26$ for every $\mathbf{C}(i, j, k)$ in $\mathbf{Z}^{3}$. If we make a comparison between $\mathbf{B}_{6}^{+}(i, j, k)$ and $\mathbf{B}_{18}^{+}(i, j, k)$ in Table 2 , we see

$$
\mathbf{B}_{6}^{+}(i, j, k) \supset \mathbf{B}_{18}^{+}(i, j, k)
$$


Table 3. The classification of all points in $\mathbf{I}^{+}$into two types with respect to each configuration of Table 2: a set of black and gray points which are included in $\mathbf{B}_{26}^{+}$and a set of white points which are not included in $\mathbf{B}_{26}^{+}$

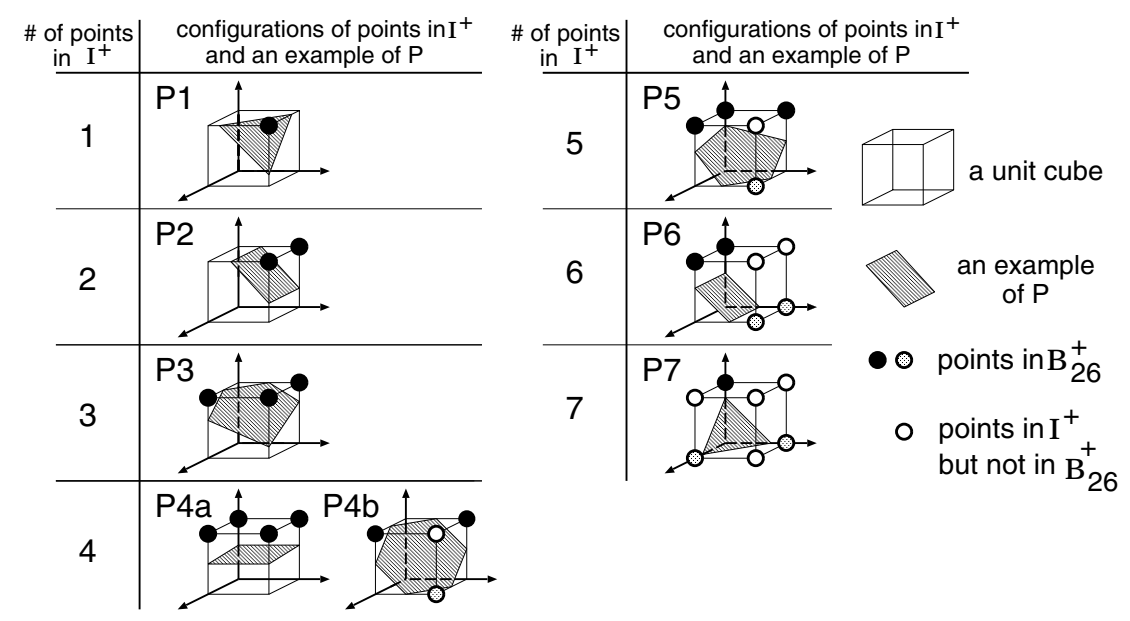

for configurations P4b, P5, P6 and P7, otherwise we obtain

$$
\mathbf{B}_{6}^{+}(i, j, k)=\mathbf{B}_{18}^{+}(i, j, k) .
$$

Between $\mathbf{B}_{18}^{+}(i, j, k)$ and $\mathbf{B}_{26}^{+}(i, j, k)$, we see

$$
\mathbf{B}_{18}^{+}(i, j, k)=\mathbf{B}_{26}^{+}(i, j, k)
$$

for any configuration in Table 2, even if $\partial \mathbf{I}_{18}^{+}(i, j, k)$ and $\partial \mathbf{I}_{26}^{+}(i, j, k)$ are different for P5 and P6. From (15), (16) and (17), we see that (13) holds.

We then prove the next theorem.

Theorem 2. For any $\mathbf{P}$, the equality relations

$$
\mathbf{N P}=\mathbf{B}_{18}^{+}=\mathbf{B}_{26}^{+}
$$

hold.

Proof. Let us consider $\mathbf{P}$ such that $0 \leq a \leq b \leq c, c>0$. In this case $w=c$. From (2) we obtain

$$
\mathbf{N P}=\left\{(x, y, z) \in \mathbf{Z}^{3}:-\frac{a}{c} x-\frac{b}{c} y-\frac{d}{c} \leq z<-\frac{a}{c} x-\frac{b}{c} y-\frac{d}{c}+1\right\} .
$$

For every point $\boldsymbol{x}=(x, y, z)$ in $\mathbf{N P}$, if we set a point $\boldsymbol{c}_{\boldsymbol{x}} \in \mathbf{P}$ such that

$$
\boldsymbol{c}_{\boldsymbol{x}}=\left(x, y,-\frac{a}{c} x-\frac{b}{c} y-\frac{d}{c}\right),
$$


then we see that

$$
0 \leq\left|\boldsymbol{x}-\boldsymbol{c}_{\boldsymbol{x}}\right|<1
$$

from (19). Since $\mathbf{B}_{18}^{+}=\mathbf{B}_{26}^{+}$from Lemma 1, we will show that every $\boldsymbol{x} \in \mathbf{B}_{26}^{+}$ satisfies (21) and no point in $\mathbf{Z}^{3} \backslash \mathbf{B}_{26}^{+}$satisfies (21) to prove this theorem. Let us consider a cubic region $\mathbf{C}(i, j, k)$ of $(10)$. Table 2 gives all configurations of points in $\mathbf{I}^{+}$and $\left(\mathbf{I}^{+}\right)^{\prime}$ for a $\mathbf{C}(i, j, k)$. All black points in Table 2 are classified into black, gray and white points in Table 3 . All black points in Table 3 apparently satisfy (21). Let us consider the case $a>0$. For each gray point $\boldsymbol{x}=(x, y, z)$, if we set two points in $\mathbf{P}$ such as

$$
\boldsymbol{a}_{\boldsymbol{x}}=\left(-\frac{b}{a} y-\frac{c}{a} z-\frac{d}{a}, y, z\right)
$$

and

we obtain

$$
\boldsymbol{b}_{\boldsymbol{x}}=\left(x,-\frac{a}{b} x-\frac{c}{b} z-\frac{d}{b}, z\right)
$$

$\left|\boldsymbol{x}-\boldsymbol{a}_{\boldsymbol{x}}\right| \geq\left|\boldsymbol{x}-\boldsymbol{b}_{\boldsymbol{x}}\right| \geq\left|\boldsymbol{x}-\boldsymbol{c}_{\boldsymbol{x}}\right|$

since $\left|\boldsymbol{x}-\boldsymbol{a}_{\boldsymbol{x}}\right|:\left|\boldsymbol{x}-\boldsymbol{b}_{\boldsymbol{x}}\right|:\left|\boldsymbol{x}-\boldsymbol{c}_{\boldsymbol{x}}\right|=1 / a: 1 / b: 1 / c$ from Lemma 2 in Appendix and $0<a \leq b \leq c$. We then see in Table 3 that every gray point $\boldsymbol{x}$ satisfies

$$
\left|\boldsymbol{x}-\boldsymbol{a}_{\boldsymbol{x}}\right|<1 \text { or }\left|\boldsymbol{x}-\boldsymbol{b}_{\boldsymbol{x}}\right|<1
$$

and from (24) we obtain (21). In the case $a=0$, we will have only P2, P4a and $\mathrm{P} 6$ for configurations of $\mathbf{C}(i, j, k)$ in Table 3, and gray points exist only in $\mathrm{P} 6$. If $b>0$, we set $\boldsymbol{b}_{\boldsymbol{x}}$ and have the second inequation of (25). Thus, we also obtain (21). If $b=0$, we will have only $\mathrm{P} 4 \mathrm{a}$ in which no gray point exists. Obviously, no white point in Table 3 satisfies (21). From a comparison between Tables 2 and 3 , we see that a set of black and gray points in Table 3 is equal to a set of points of $\mathbf{B}_{26}^{+}$in Table 2. Thus, we have (18).

Theorem 2 indicates that either of $\partial \mathbf{I}_{18}^{+}$or $\partial \mathbf{I}_{26}^{+}$is a triangulation of $\mathbf{N P}$ in the aspect of combinatorial topology. From Lemma 1 and Theorem 2, we obtain the next corollary.

Corollary 1. For any $\mathbf{P}$, the inclusion relation

$$
\mathbf{N P} \subseteq \mathbf{B}_{6}^{+}
$$

holds.

If $\mathbf{N P}=\mathbf{B}_{6}^{+}$, we say that $\partial \mathbf{I}_{6}^{+}$is also a triangulation of $\mathbf{N P}$, but if $\mathbf{N P} \subset \mathbf{B}_{6}^{+}$, it is not obviously. If we define a naive plane such that

$$
\mathbf{N P}^{-}=\left\{(x, y, z) \in \mathbf{Z}^{3}:-w<a x+b y+c z+d \leq 0\right\}
$$

instead of NP and set

$$
\mathbf{B}_{m}^{-}=\underset{[a] \in \partial \mathbf{I}_{m}^{-}}{\cup}[a]
$$

for $m=6,18,26$, then the following corollary is derived. 
Corollary 2. For any $\mathbf{P}$, the relations

$$
\mathbf{N P}^{-}=\mathbf{B}_{18}^{-}=\mathbf{B}_{26}^{-}
$$

and

$$
\mathrm{NP}^{-} \subseteq \mathbf{B}_{6}^{-}
$$

hold.

Equation (29) in Corollary 2 indicates that either of $\partial \mathbf{I}_{18}^{-}$or $\partial \mathbf{I}_{26}^{-}$is a triangulation of $\mathbf{N P}^{-}$in the aspect of combinatorial topology.

\section{Properties of Naive Planes as Discrete Planar Surfaces}

In this section, we discuss the local configurations of discrete simplexes in $\partial \mathbf{I}_{6}^{+}$, $\partial \mathbf{I}_{18}^{+}$and $\partial \mathbf{I}_{26}^{+}$with respect to $\mathbf{P}$. From Theorem 2 and the properties of local point configurations of NP which have been introduced in $[4,5,6,7]$, we can derive the combinatorial properties of $\partial \mathbf{I}_{6}^{+}, \partial \mathbf{I}_{18}^{+}$and $\partial \mathbf{I}_{26}^{+}$; they are summarized in Propositions 1 to 5 . Let us consider the configurations of discrete simplexes in the parts of $\partial \mathbf{I}_{m}^{+}$for $m=6,18,26$ which project on the coordinate plane $z=0$ as a rectangle whose sizes are $\lambda \times \mu$.

Proposition 1. In the case of $\lambda=\mu=2$, there exist five different configurations in $\mathbf{N P} s$ and the corresponding configurations of discrete simplexes for $\partial \mathbf{I}_{m}^{+}$, $m=6,18,26$, are shown in Fig. 1 with respect to any $\mathbf{P}$ such that $0 \leq a \leq b \leq c$, $c>0$.

Proposition 2. At most four different configurations of discrete simplexes for $\lambda=\mu=2$ are contained in a $\partial \mathbf{I}_{m}^{+}, m=6,18,26$.

Proposition 3. In the case of $\lambda=\mu=3$, there exist 40 different configurations in $\mathbf{N P} s$ and the corresponding configurations of discrete simplexes for $\partial \mathbf{I}_{m}^{+}, m=$ 6,18,26, are shown in Figs. 2, 3 and 4, respectively, with respect to any $\mathbf{P}$ such that $0 \leq a \leq b \leq c, c>0$.

Proposition 4. At most nine different configurations of discrete simplexes for $\lambda=\mu=3$ are contained in a $\partial \mathbf{I}_{m}^{+}, m=6,18,26$.
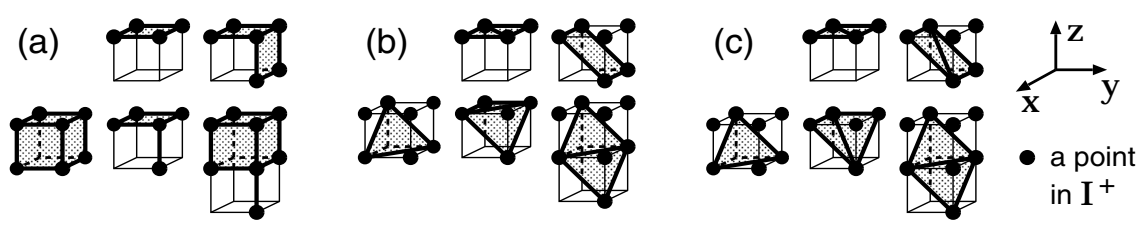

Fig. 1. All five configurations of discrete simplexes in $\partial \mathbf{I}_{6}^{+}$(a), $\partial \mathbf{I}_{18}^{+}$(b) and $\partial \mathbf{I}_{26}^{+}$ (c), whose projections on plane $z=0$ lie on the $2 \times 2$ square grids 


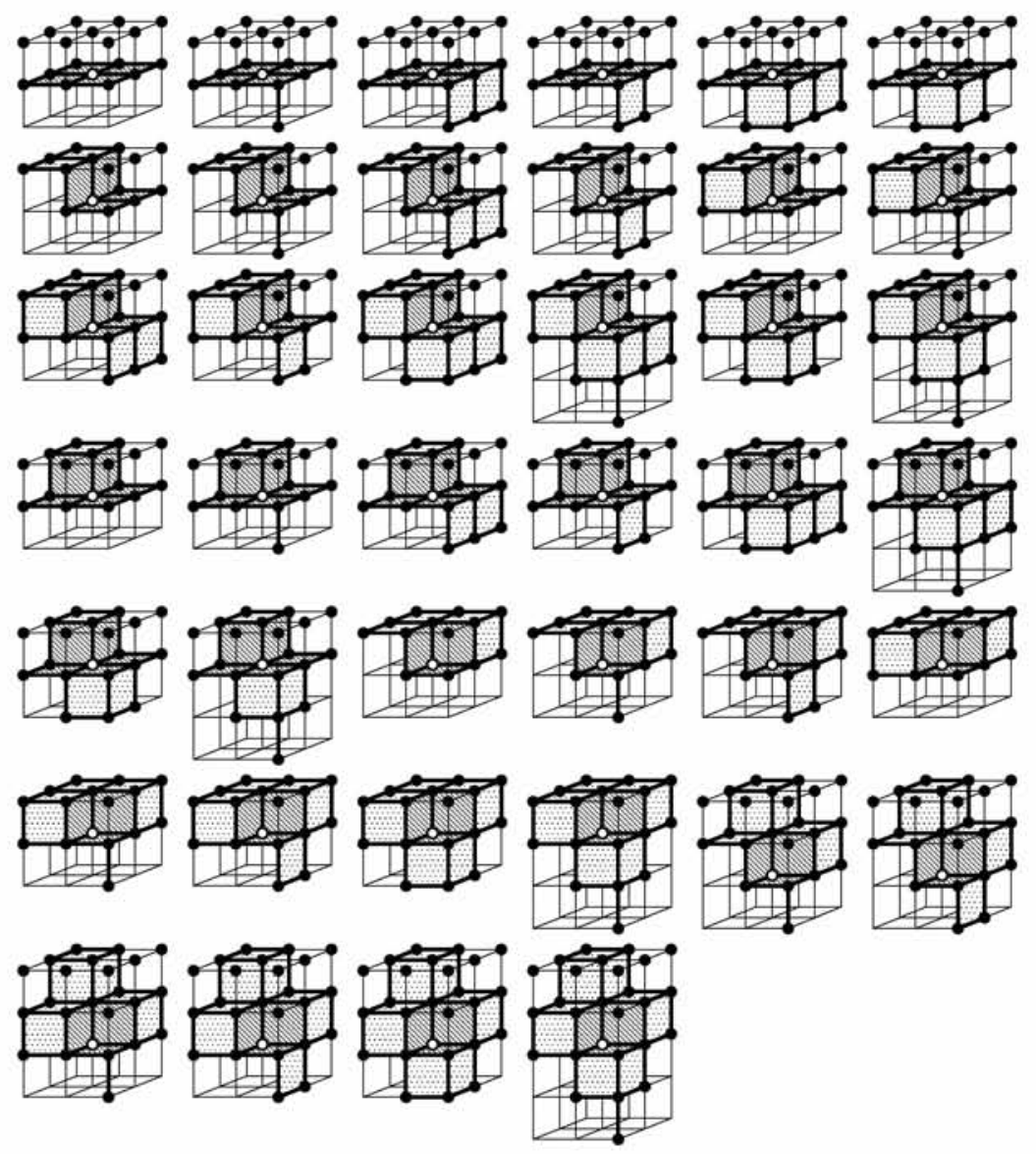

Fig. 2. All 40 configurations of discrete simplexes in $\partial \mathbf{I}_{6}^{+}$whose projections on plane $z=0$ lie on the $3 \times 3$ square grids. The star of each white point is also shown as discrete simplexes with diagonal lines in the figures

Propositions 1 to 4 give the coexistence of adjacent 2-simplexes in a $\partial \mathbf{I}_{m}^{+}$, $m=6,18,26$. There are actually two simplicial configurations for $\mathrm{P} 4 \mathrm{a}$ in $\partial \mathbf{I}_{18}^{+}$ and $\partial \mathbf{I}_{26}^{+}$, and for $\mathrm{P} 6$ in $\partial \mathbf{I}_{26}^{+}$as shown in Fig. 5. For each 0 -simplex $[\boldsymbol{x}] \in \partial \mathbf{I}_{m}^{+}$, we can define the star such that

$$
\sigma\left([\boldsymbol{x}]: \partial \mathbf{I}_{m}^{+}\right)=\left\{[a] \in \partial \mathbf{I}_{m}^{+}:[\boldsymbol{x}] \in \operatorname{face}([a])\right\} .
$$

In Figs. 3 and 4, we choose one of the configurations in Fig. 5 for P4a or P6 so that the number of 2-simplexes in the star of a white point $[\boldsymbol{x}]$ becomes as small as possible. The projection of $\sigma\left([\boldsymbol{x}]: \partial \mathbf{I}_{m}^{+}\right)$on the coordinate plane $z=0$ is in a square whose size is $3 \times 3$ if $0 \leq a \leq b \leq c$ and $c>0$. From this fact, we also derive the following proposition. 

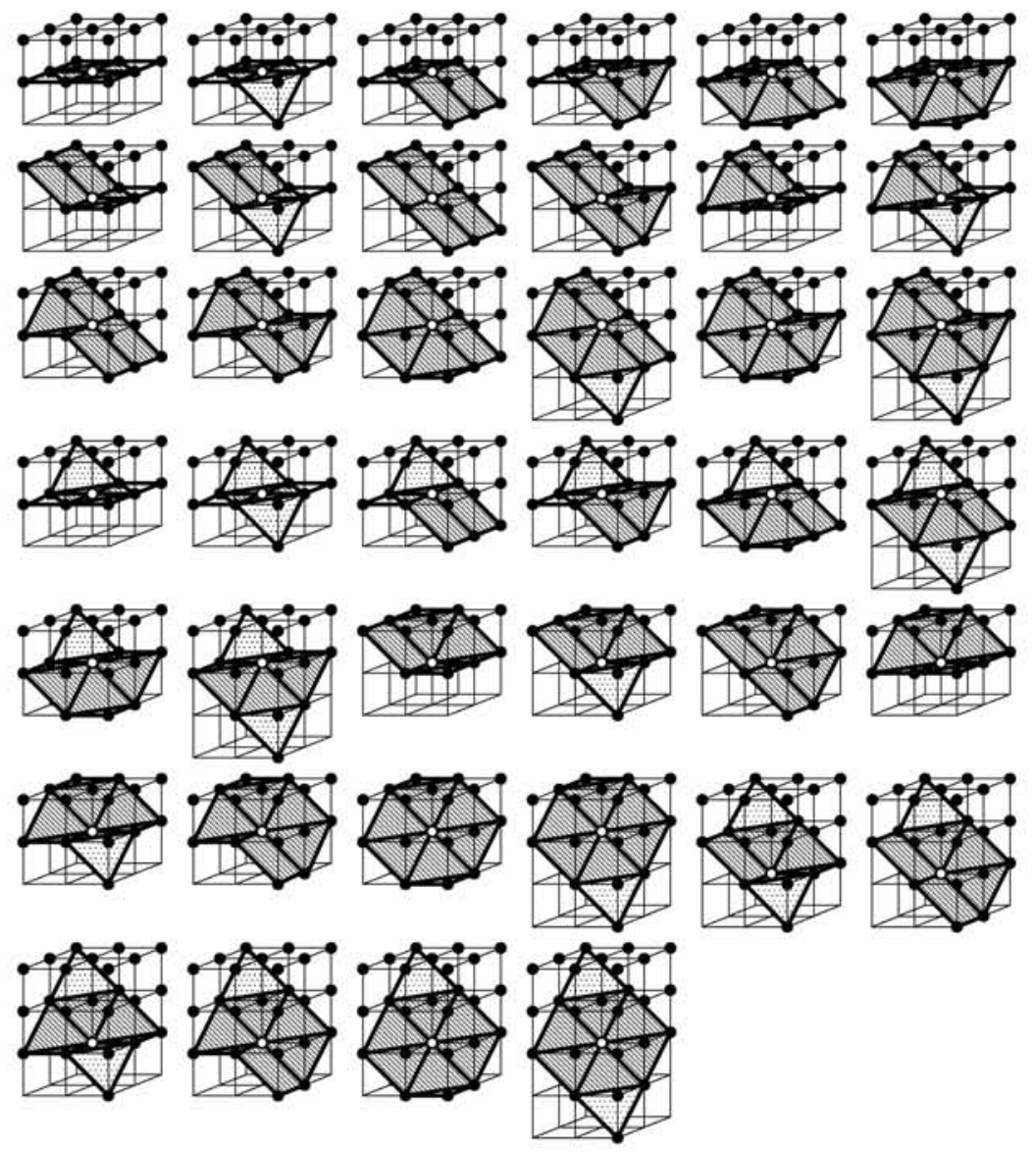

Fig. 3. All 40 configurations of discrete simplexes in $\partial \mathbf{I}_{18}^{+}$whose projections on plane $z=0$ lie on the $3 \times 3$ square grids. The star of each white point is also shown as discrete simplexes with diagonal lines in the figures

Proposition 5. Any discrete planar surface $\partial \mathbf{I}_{m}^{+}$for $m=6,18,26$ consists of 2-simplexes and their faces so that every 0 -simplex $[\boldsymbol{x}] \in \partial \mathbf{I}_{m}^{+}$has one of the stars whose configurations are illustrated in Figs. 2, 3 and 4, respectively.

We see that the equivalent simplicial configurations of a star can appear in different simplicial configurations each of which projects on the coordinate plane $z=0$ as a $3 \times 3$ square in Figs. 2, 3 and 4 . Thus, the total number of different configurations of discrete simplexes of a star will be less than 40, i.e. 4, 18 and 23 configurations for the 6-, 18- and 26-neighborhood systems, respectively. The similar results of local configurations for $\partial \mathbf{I}_{6}^{+}$are also presented in $[1,4]$. 


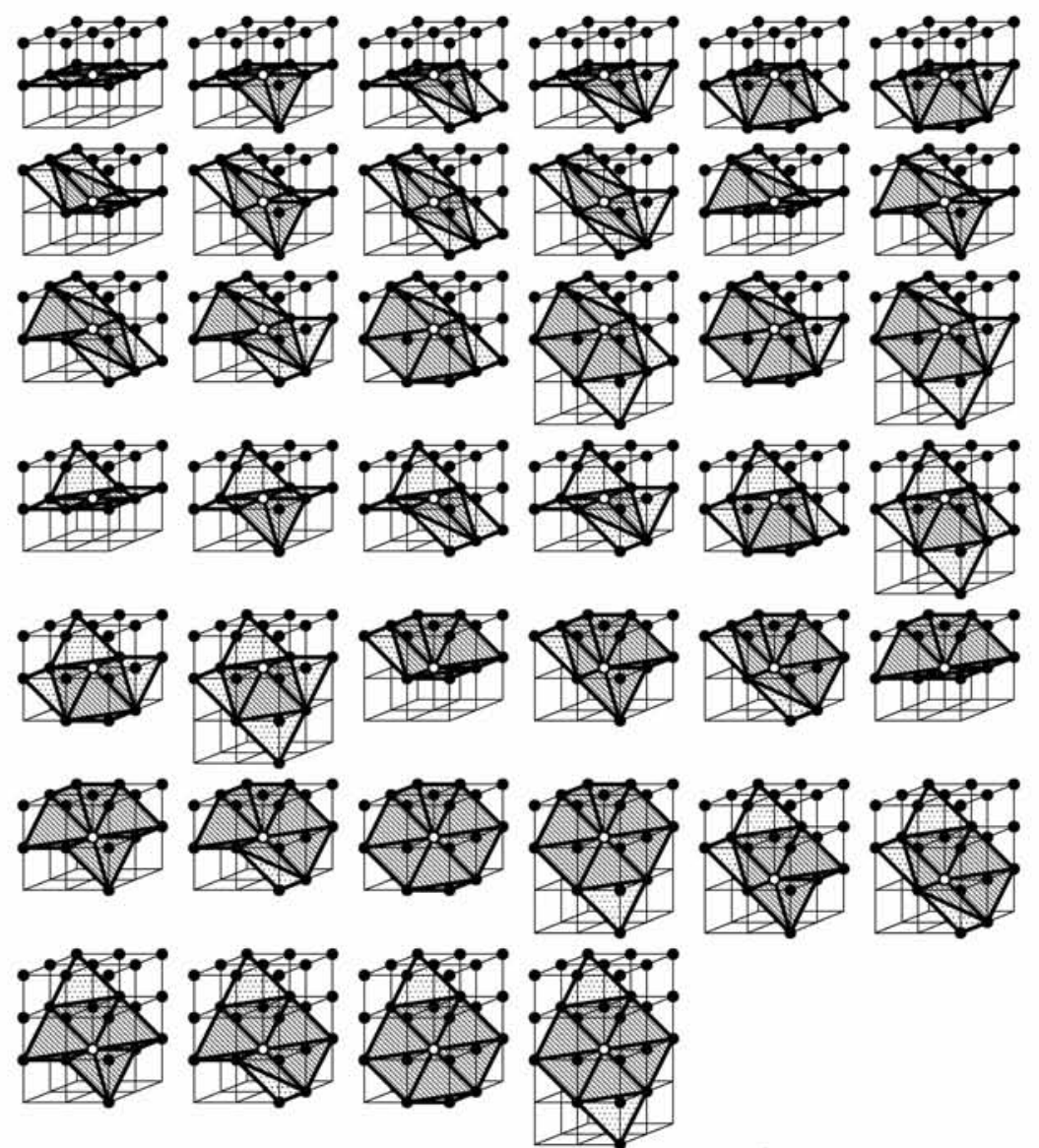

Fig. 4. All 40 configurations of discrete simplexes in $\partial \mathbf{I}_{26}^{+}$whose projections on plane $z=0$ lie on the $3 \times 3$ square grids. The star of each white point is also shown as discrete simplexes with diagonal lines in the figures

\section{Conclusions}

In this paper, we first proved that $\mathbf{B}_{18}^{+}$and $\mathbf{B}_{26}^{+}$are equal to NP. From the equality relations, we see that $\partial \mathbf{I}_{m}^{+}$for $m=18,26$ is a triangulation of NP in the sense of combinatorial topology. Since $\partial \mathbf{I}_{m}^{+}$consists of discrete simplexes, we described the local properties of $\partial \mathbf{I}_{m}^{+}$by using configurations of discrete simplexes instead of those of lattice points, such as the coexistence of adjacent 2 -simplexes and the configuration of discrete simplexes of a star in a $\partial \mathbf{I}_{m}^{+}$. If we set $w=|a|+|b|+|c|$ in (2), we obtain standard planes with respect to $\mathbf{P}$ of (1), instead of naive planes [4]. It is our future work to clarify the relations between standard planes and $\partial \mathbf{I}_{m}^{+}, m=6,18,26$. A part of this work was supported by JSPS Grant-in-Aid for Encouragement of Young Scientists (12780207). 
(a)

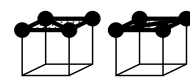

(b)

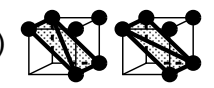

Fig. 5. Two simplicial configurations of $\mathrm{P} 4 \mathrm{a}$ for $m=18,26$ (a) and $\mathrm{P} 6$ for $m=26(\mathrm{~b})$

\section{References}

1. Imiya, A., Eckhardt, U.: The Euler Characteristics of Discrete Objects and Discrete Quasi-Objects. Computer Vision and Image Understanding 753 (1999) 307-318 249, 258

2. Borgefors, G., Nyström, I., di Baja, G. S.: Computing skeletons in three dimensions. Pattern Recognition 32 (1999) 1225-1236 249

3. Andres, E.: Le Plan Discret. In Proceedings of 3e Colloque Géométrie discète en imagerie: fondements et applications. Strasbourg (1993) 45-61 249

4. Françon, J.: Sur la topologie d'un plan arithmétique. Theoretical Computer Science 156 (1996) 159-176 249, 256, 258, 259

5. Reveillès, J. P.: Combinatorial Pieces in Digital Lines and Planes. In Vision Geometry III. Proceedings of SPIE, Vol. 2573 (1995) 23-34 249, 250, 256

6. Françon, J., Schramm, J. M., Tajine, M.: Recognizing arithmetic straight lines and planes. In Discrete Geometry for Computer Imagery. LNCS 1176 Springer-Verlag, Berlin, Heidelberg (1996) 141-150 249, 256

7. Debled-Renesson, I.: Etude et reconnaissance des droites et plans discrets. $\mathrm{PhD}$ thesis, University of Louis Pasteur (1995) 249, 256

8. Kenmochi, Y.: Discrete Combinatorial Polyhedra: Theory and Application. Doctoral thesis, Chiba University (1998) 249, 251, 252

9. Kenmochi, Y., Imiya, A.: On Combinatorial Properties of Discrete Planar Surfaces. In Proceedings of International Conference on Free Boundary Problems: Theory and Applications. Vol. 2. Gakkotosho, Tokyo (2000) 255-272. 249, 253

10. Aleksandrov, P. S.: Combinatorial Topology. Vol. 1. Graylock Press, Rochester, New York (1956) 249, 250

\section{Appendix: Lemma 2}

Let us consider $\mathbf{P}$ of (1) such that $a, b, c>0$. For each point $\boldsymbol{p} \in \mathbf{I}^{+} \backslash \mathbf{P}$ such that $\boldsymbol{p}=(s, t, u)$, we set three planes such as

$$
\begin{aligned}
& \mathbf{S}=\left\{(x, y, z) \in \mathbf{R}^{3}: x=s\right\}, \\
& \mathbf{T}=\left\{(x, y, z) \in \mathbf{R}^{3}: y=t\right\}, \\
& \mathbf{U}=\left\{(x, y, z) \in \mathbf{R}^{3}: z=u\right\} .
\end{aligned}
$$

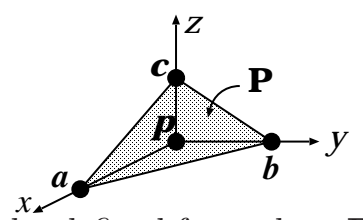

Fig. 6. Three points $\boldsymbol{a}, \boldsymbol{b}$ and $\boldsymbol{c}$ defined for a plane $\mathbf{P}$ and a point $\boldsymbol{p}$ which is not in $\mathbf{P}$ 
Let $\boldsymbol{a}, \boldsymbol{b}$ and $\boldsymbol{c}$ be the intersection points of $\mathbf{P}, \mathbf{T}$ and $\mathbf{U}, \mathbf{P}, \mathbf{S}$ and $\mathbf{U}$, and $\mathbf{P}, \mathbf{S}$ and $\mathbf{T}$, respectively, as illustrated in Fig. 6. Then the next lemma is derived.

Lemma 2. For any $\boldsymbol{p} \in \mathbf{I}^{+} \backslash \mathbf{P}$, we obtain

$$
|\boldsymbol{p}-\boldsymbol{a}|:|\boldsymbol{p}-\boldsymbol{b}|:|\boldsymbol{p}-\boldsymbol{c}|=1 / a: 1 / b: 1 / c
$$

where $a, b, c>0$.

Proof. The equation of the line which is the intersection of $\mathbf{P}$ and $\mathbf{U}$ is given by $a x+b y+c u+d=0$. Thus, the slope of the line in $\mathbf{U}$ is given by

$$
\frac{|\boldsymbol{p}-\boldsymbol{b}|}{|\boldsymbol{p}-\boldsymbol{a}|}=\frac{a}{b} \text {. }
$$

Similarly, the slopes of the intersection lines between $\mathbf{P}$ and $\mathbf{T}$, and $\mathbf{P}$ and $\mathbf{S}$, are respectively given by

$$
\frac{|\boldsymbol{p}-\boldsymbol{c}|}{|\boldsymbol{p}-\boldsymbol{b}|}=\frac{b}{c} \quad \text { and } \quad \frac{|\boldsymbol{p}-\boldsymbol{a}|}{|\boldsymbol{p}-\boldsymbol{c}|}=\frac{c}{a} .
$$

From (36) and (37), we obtain (35). 